\title{
Pressure suppresses hepatocellular glycogen synthesis through activating the $\mathrm{p53} /$ Pten pathway
}

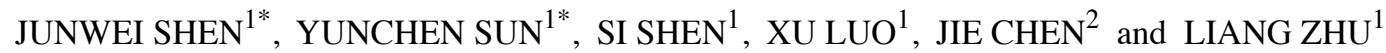 \\ ${ }^{1}$ Department of Gastroenterology, Changzheng Hospital, Second Military Medical University, Shanghai 200003; \\ ${ }^{2}$ Department of Hematology, Changhai Hospital, Second Military Medical University, Shanghai 200168, P.R. China
}

Received August 4, 2018; Accepted March 25, 2019

DOI: $10.3892 / \mathrm{mmr} .2019 .10177$

\begin{abstract}
Portal hypertension is the primary cause of complications in patients with chronic liver diseases, and markedly impacts metabolism within the nervous system. Until recently, the role of portal hypertension in hepatocellular metabolism was unclear. The present study demonstrated that an increase in extracellular pressure significantly decreased hepatocellular glycogen concentrations in HepG2 and HL-7702 cells. In addition, it reduced glycogen synthase activity, by inhibiting the phosphorylation of glycogen synthase 1. RNA-seq analysis revealed that mechanical pressure suppressed glycogen synthesis by activating the p53/phosphatase and tensin homolog pathway, further suppressing glycogen synthase activity. The present study revealed an association between mechanical pressure and hepatocellular glycogen metabolism, and identified the regulatory mechanism of glycogen synthesis under pressure.
\end{abstract}

\section{Introduction}

Numerous biochemical factors influence the extracellular microenvironment, including hormones and small molecules. Additionally, biophysical factors, including mechanical pressure, are notable components in the maintenance of the extracellular microenvironment; in particular, muscles, blood vessels, bone and dental tissues are under high degrees of pressure. The mechanical microenvironment serves a prominent regulatory role in cell proliferation, growth,

Correspondence to: Dr Liang Zhu, Department of Gastroenterology, Changzheng Hospital, Second Military Medical University, 415 Fengyang Road, Shanghai 200003, P.R. China

E-mail: czzhuliang@smmu.edu.cn

Dr Jie Chen, Department of Hematology, Changhai Hospital, Second Military Medical University, 168 Changhai Road, Shanghai 200168, P.R. China

E-mail: chenjiedoctor@163.com

*Contributed equally

Key words: pressure, glycogen synthesis, p53, Pten differentiation and metabolism, and it has been reported that external factors control mitotic spindle positioning to regulate cell proliferation (1). Notably, Lesman et al (2) demonstrated that contractile forces regulate cell division in three-dimensional environments. The effects of biomechanical factors are more profound on motor system-associated cells, including bone and muscle cells; moderate mechanical pressure regulates the proliferation and differentiation of osteoblasts (3). Furthermore, mechanical loading synergistically increases trabecular bone volume and improves the mechanical properties of mice (4). Fluid shear stress inhibits osteoblast apoptosis via the extracellular signal-regulated kinase 5 signaling pathway (5). It has also been reported that biophysical factors regulate the pluripotency of stem cells; specific pressures (300 and $600 \mathrm{~Pa}$ ) accelerate the induction of pluripotency in defined three-dimensional microenvironments (6). Additional studies on the role of mechanical factors in cell function have focused on physiology $(7,8)$. However, the number of studies on the role of mechanical factors in pathological conditions, such as hepatic portal hypertension, is limited.

Due to the absence of valves in the portal vein, retrograde blood flow cannot be prevented. When pre-hepatic, intrahepatic or post-hepatic obstruction occurs, retrograde flow is possible and may result in portal hypertension (9). Portal hypertension is the primary cause of complications in patients with chronic liver diseases (10). Such complications include esophageal and gastric varices, variceal bleeding, ascites, spontaneous bacterial peritonitis, splenomegaly and hepatic encephalopathy. Portal hypertension also has notable effects on metabolism (11-13); hepatic encephalopathy, a serious complication of portal hypertension, is a central nervous system dysfunction resulting from a metabolic disorder $(14,15)$. Previous studies have revealed that portal hypertension inhibits the activity of cytochrome $c$ oxidase in the nerve cells of the dentate gyrus and the basolateral, medial, lateral and central amygdala, thereby affecting the brain's memory function $(14,16,17)$. Portal hypertension markedly influences metabolism within the nervous system; however, its effects on hepatocyte metabolism remain unclear.

Blood glucose equilibrium is one of the most notable metabolic balances of the human body, and the liver is the primary organ for carbohydrate metabolism and blood glucose maintenance. The liver maintains blood glucose levels by regulating 
glycogen synthesis and glycogenolysis (18). These processes are tightly controlled by various factors. In particular, insulin, a principle regulator of blood glucose, stimulates glycogen synthesis and inhibits glycogenolysis (19). Further studies have revealed that insulin promotes glycogen synthesis through binding of its receptor and activating the downstream protein kinase $\mathrm{B} / \mathrm{glycogen}$ synthase kinase $3 \beta$ (Akt/GSK3 $\beta$ ) pathway (20). Numerous studies have been conducted on the regulation of glycogen synthesis in the liver (21-23). However, in pathological conditions, the mechanism underlying regulation of glycogen synthesis remains unidentified. In the present study, it was revealed that mechanical pressure inhibited hepatocellular glycogen synthesis, and the regulatory mechanism of glycogen synthesis under pressure was identified.

\section{Materials and methods}

Cell culture. The HL-7702 human hepatocyte cell line and HepG2 hepatoblastoma cell line were purchased from the Cell Bank of the Type Culture Collection of the Chinese Academy of Sciences, Shanghai Institute of Cell Biology, Chinese Academy of Sciences. The cells were cultured in high-glucose Dulbecco's modified Eagle's medium (Gibco; Thermo Fisher Scientific, Inc) supplemented with $10 \%$ FBS (Gibco; Thermo Fisher Scientific, Inc) and 1\% penicillin/streptomycin (Hyclone; GE Healthcare Life Sciences). Cells were maintained in a humidified atmosphere containing $5 \% \mathrm{CO}_{2}$ at $37^{\circ} \mathrm{C}$ in a cell incubator (Thermo Fisher Scientific, Inc.).

Pressure loading. The pressure-loading apparatus was set up as described previously, with some modifications $(24,25)$, namely, the apparatus were composed of an air-tight steel chamber with inlet and outlet ports. To generate pressure, compressed helium gas was released into the chamber. The pressure was confirmed by a sphygmomanometer through a tube connected to the outlet. Cells used for experiments were placed in the chamber inside the incubator. After culturing at $37^{\circ} \mathrm{C}$ for $24 \mathrm{~h}$, the cells were exposed to pressure between 0 and $15 \mathrm{mmHg}$ for $24 \mathrm{~h}$ at $37^{\circ} \mathrm{C}$ for further experiments.

Glycogen analysis assay. For the Periodic Acid-Schiff (PAS) assay, the cells were seeded in 24-well plates at a density of $1 \times 10^{4}$ cells/well, and were cultured for $24 \mathrm{~h}$. The cells were exposed to different pressures and were stained using the Glycogen PAS Staining kit (cat. no. G1360; Beijing Solarbio Science \& Technology Co., Ltd.) according to the manufacturer's protocol. The staining data were analyzed with Image-Pro Plus 6.0 software (Media Cybernetics, Inc.). The cell images were captured using a fluorescence microscope (Nikon ECLIPSE Ti; Nikon Corporation).

To further analyze glycogen concentration, cells were seeded into 24 -well plates at a density of $1 \times 10^{4}$ cells/well, cultured for $24 \mathrm{~h}$ and exposed to $0-15 \mathrm{mmHg}$ pressure. The cells were washed with PBS and the glycogen content was determined using the Glycogen Content Detection kit (cat. no. BC0345; Beijing Solarbio Science \& Technology Co., Ltd.) according to the manufacturer's protocol. Absorbance was determined at $620 \mathrm{~nm}$ using a SpectraMax M5 spectrophotometer (Thermo Fisher Scientific, Inc.).
Cell Counting kit (CCK)- 8 assay. The cells were seeded in 96-well plates at a density of $1 \times 10^{3}$ cells/well. Subsequently, the cells were exposed to different pressures for various durations and $10 \mu \mathrm{l}$ CCK-8 reagent (Dojindo Molecular Technologies, Inc.) was added to each well at $37^{\circ} \mathrm{C}$ for $1 \mathrm{~h}$. Finally, absorbance was determined at $450 \mathrm{~nm}$ using a SpectraMax M5 spectrophotometer.

Apoptosis analysis. Cell apoptosis was detected using an Annexin V-FITC/propidium iodide (PI) cell apoptosis kit (cat. no. KGA108-1; KeyGen Biotech Co., Ltd.) according to the manufacturer's protocol. Briefly, cells reseeded at a density of $1 \times 10^{5}$ cells/well in a 6-well plate were exposed to different pressures. Subsequently, the cells were washed with ice-cold PBS, collected in a $1.5 \mathrm{ml}$ Eppendorf tube, and treated with Annexin V-FITC/PI for $10 \mathrm{~min}$ at room temperature according to the manufacturer's protocol. The cells were then analyzed using a flow cytometer (BD FACSVerse; BD Biosciences). Data were analyzed using FlowJo V10 Analysis software (FlowJo LLC).

Reverse transcription-quantitative PCR (RT-qPCR) and $R N A$-seq. Total RNA was isolated using TRIzol ${ }^{\circledR}$ reagent (Invitrogen; Thermo Fisher Scientific, Inc.) according to the manufacturer's protocol. For each sample, 500 ng RNA was reverse transcribed to cDNA using a Prime-Script RT reagent kit (cat. no. RR037A; Takara Biotechnology Co.,Ltd.) according to the manufacturer's protocol. The obtained cDNA was amplified using the Takara Ex Taq PCR kit (Takara Biotechnology Co., Ltd.) and qPCR amplification was conducted using the Stratagene Mx3000 QPCR system (Stratagene; Agilent Technologies, Inc.) and analyzed via the $2^{-\Delta \Delta C q}$ method (26). The thermocycling conditions were as follows: $95^{\circ} \mathrm{C}$ for $1 \mathrm{~min}$, and 40 cycles of $95^{\circ} \mathrm{C}$ for $10 \mathrm{sec}$ and $60^{\circ} \mathrm{C}$ for $30 \mathrm{sec}$. A melting curve was subsequently generated. The primer sequences used in these assays are shown in Table I.

For RNA-seq, 500 ng extracted total RNA from each sample was used for library construction. Libraries were constructed using Truseq RNA Sample Preparation kit V2 (Illumina, Inc; San Diego, USA) according to the manufacturer's protocol. Adapters with index sequences were attached to the libraries. After the average length of the libraries was confirmed, the concentration of each library was adjusted to $10 \mathrm{nM}$. Sequencing was performed for $100 \mathrm{bp}$ using HiSeq2500 (Illumina, Inc.), with the single-read method. Mapping, data normalization and statistical analyses were performed using CLC Genomics Workbench (v. 8.5.1; Qiagen, Inc.). For volcano plotting, gene expression levels were averaged over the replicates for each cell line post-normalization and the cell lines were compared by calculating the fold change (FC). FC was defined as the ratio between the averages and P-values were determined for each gene expressed. In volcano plots, FC was plotted along the $\mathrm{x}$-axis and P-value along the $y$-axis. Significantly altered genes were defined as those with thresholds of $\mathrm{FC}>2$ and $\mathrm{P}<0.01$.Gene set enrichment analysis (GSEA) was performed at www.broadinstitute.org/gsea.

Western blot analysis. Cultured cells were lysed in strong RIPA buffer (cat. no. R0010; Beijing Solarbio Science \& Technology Co., Ltd.) containing Halt Protease Inhibitor 
Table I. Primer sequences used in reverse transcriptionquantitative PCR.

\begin{tabular}{ll} 
Gene & \multicolumn{1}{c}{ Sequence (5'-3') } \\
\hline Gys1-F & ACAACCTGGAGAACTTCAAC \\
Gys1-R & ATCTGGGACACAGTAGTGAA \\
Gsk3 $\beta-F$ & CTCCTCATGCTCGGATTCA \\
Gsk3 $\beta-\mathrm{R}$ & TGCAGAAGCAGCATTATTGG \\
Agl-F & CATTTAATAGAGAAAAATTC \\
Agl-R & CATGATTATCAGCACCAACACGT \\
G6pc3-F & GATGCCTAGCCTGGCTTATT \\
G6pc3-R & CAGGACAGCGCCAGTTATTA \\
p53-F & CAAACCCCTGGTTTAGCACTTC \\
p53-R & TGTCCTTCCTGGAGCGATCT \\
Pten-F & CCGAAAGGTTTTGCTACCATTCT \\
Pten-R & AAAATTATTTCCTTTCTGAGCATTCC \\
Akt-F & GCAGCACGTGTACGAGAAGA \\
Akt-R & GGTGTCAGTCTCCGACGTG \\
Pygl-F & TGCCCGGCTACATGAATAACA \\
Pygl -R & TGTCATTGGGATAGAGGACCC \\
Gapdh-F & CCCATCACCATCTTCCAGGAGC \\
Gapdh-R & CAGTGAGCTTCCCGTTCAGC \\
\hline
\end{tabular}

F, forward; R, reverse; Agl, amylo- $\alpha-1$, 6-glucosidase, 4-alpha-glucanotransferase; Akt, protein kinase $\mathrm{B}$; G6pc3, glucose-6-phosphatase catalytic subunit 3; Gsk3 $\beta$, glycogen synthase kinase $3 \beta$; Gys1, glycogen synthase 1; Pygl, glycogen phosphorylase $\mathrm{L}$; Pten, phosphatase and tensin homolog.

Cocktails (cat. no. 78430; Thermo Fisher Scientific, Inc.). Protein concentrations were measured using a BCA protein assay kit (cat. no. 23235; Pierce; Thermo Fisher Scientific, Inc.). Subsequently, $50 \mu \mathrm{g} /$ lane of protein was separated via 10\% SDS-PAGE and then transferred into a polyvinylidene difluoride membrane (cat. no. 03010040001; Sigma-Aldrich; Merck KGaA). The membranes were immersed in TBS-0.1\% Tween 20 (cat. no. T8220-100ml; Beijing Solarbio Science \& Technology Co., Ltd.) solution and blocked with 5\% skimmed milk at room temperature for $1 \mathrm{~h}$. Primary antibodies targeting glycogen synthase 1 (GYS1; 1:1,000; cat. no. ab40810; Abcam), phosphorylated (p)-GYS1 (S641; 1:1,000; cat. no. ab81230; Abcam), p53 (1:1,000; cat. no. ab26; Abcam), phosphatase and tensin homolog (Pten; 1:1,000; cat. no. ab32199; Abcam), caspase-3 (1:1,000; cat. no. ab13847; Abcam), Akt (1:1,000; cat. no. ab8805; Abcam), p-Akt (S473; 1:1,000; cat. no. ab81283; Abcam) and GAPDH (1:2,000; cat. no. sc-47724; Santa Cruz Biotechnology, Inc.) were incubated with the membranes overnight at $4^{\circ} \mathrm{C}$, followed by incubation with the horseradish peroxidase-conjugated goat anti-rabbit immunoglobulin $\mathrm{G}$ (IgG; 1:3,000; cat. no. ab97051; Abcam) and goat anti-mouse $\operatorname{IgG}(1: 3,000$; cat. no. ab6789; Abcam) for $1 \mathrm{~h}$ at room temperature. Detection and analysis of HRP was performed using the Super Signal West Pico Chemiluminescent Substrate (cat. no. 34580; Pierce; Thermo Fisher Scientific, Inc.). Quantitiative analysis was performed using Alpha View Analysis Tools (AlphaViewSA v.3.2.2 software; ProteinSimple).
Statistical analysis. Experimental results were analyzed using GraphPad Prism 5 software (GraphPad Software, Inc.). The data obtained from each group were compared with the control group. Independent samples t-tests were performed to compare the difference between two groups. The average of multiple groups was analyzed by one way analysis of variance followed by Dunnett's post hoc test. Data are presented as the mean \pm standard error of the mean of three independent experiments. $\mathrm{P}<0.05$ was considered to indicate a statistically significant difference.

\section{Results}

An increase in pressure decreases hepatocellular glycogen concentration. To evaluate the effects of extracellular pressure on hepatocellular glycogen metabolism, PAS staining was used to analyze the intracellular glycogen concentration of HepG2 cells treated with $0,5,10$ and $15 \mathrm{mmHg}$ pressure for $24 \mathrm{~h}$. A pressure of $10 \mathrm{mmHg}$ significantly decreased the intracellular glycogen concentration of HepG2 cells compared with the control group ( $0 \mathrm{mmHg}$; Fig. $1 \mathrm{~A}$ and $\mathrm{C}$ ); however, the decrease in glycogen concentration between 10 and $15 \mathrm{mmHg}$ was not substantial, indicating that the effect of pressure had reached saturation at $15 \mathrm{mmHg}$. A similar result was observed in the normal hepatic cell line HL-7702 (Fig. 1B and D), suggesting that an increase in extracellular pressure inhibited intracellular glycogen synthesis. To support this result, glycogen concentration was detected using the Glycogen Content Detection kit; when the pressure was increased, glycogen concentration gradually decreased in HepG2 and HL-7702 cells (Fig. 1E and F). The results indicated that specific pressure values decreased hepatocellular glycogen concentration, which may be associated with a decrease in glycogen synthase activity.

An increase in pressure does not affect cell viability and apoptosis. The present study detected the effects of pressure on cell viability and apoptosis. Previous data revealed that the glycogen content of hepatocytes was significantly reduced when pressure reached $10 \mathrm{mmHg}$, with no considerable difference compared with that at $15 \mathrm{mmHg}$. Therefore, 0 and $10 \mathrm{mmHg}$ pressures were employed for further study. There was no significant alteration in HepG2 or HL-7702 cell viability between the 0 and $10 \mathrm{mmHg}$ groups (Fig. 2A). Similarly, cell apoptosis was not affected by $10 \mathrm{mmHg}$ pressure (Fig. 2B and C). In addition, the protein expression levels of active caspase-3, an apoptosis marker, were not affected by pressure (Fig. 2D). These results indicated that mechanical pressure did not affect cell viability and apoptosis.

An increase in pressure suppresses glycogen synthesis by inhibiting GYS1 phosphorylation. Numerous studies have indicated that glycogen synthesis is regulated by various genes, including Gys1, GSK3b, glucose-6-phosphatase catalytic subunit 3, amylo- $\alpha-1,6$-glucosidase, 4-alpha-glucanotransferase and glycogen phosphorylase. Therefore the expression of these genes was detected in HepG2 and HL-7702 cells following treatment with different pressures. The expression levels of genes of interest were evaluated using RT-qPCR. Following treatment with 0 and $10 \mathrm{mmHg}$ pressure for $24 \mathrm{~h}$, the mRNA expression levels 
A

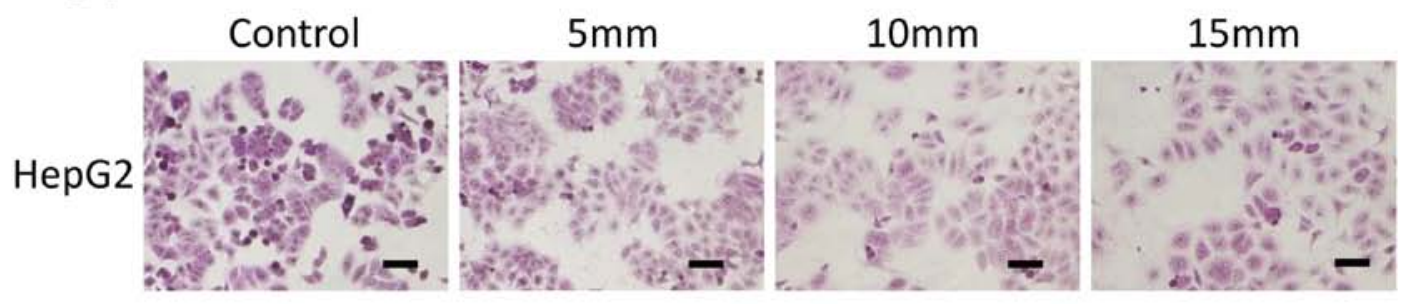

B

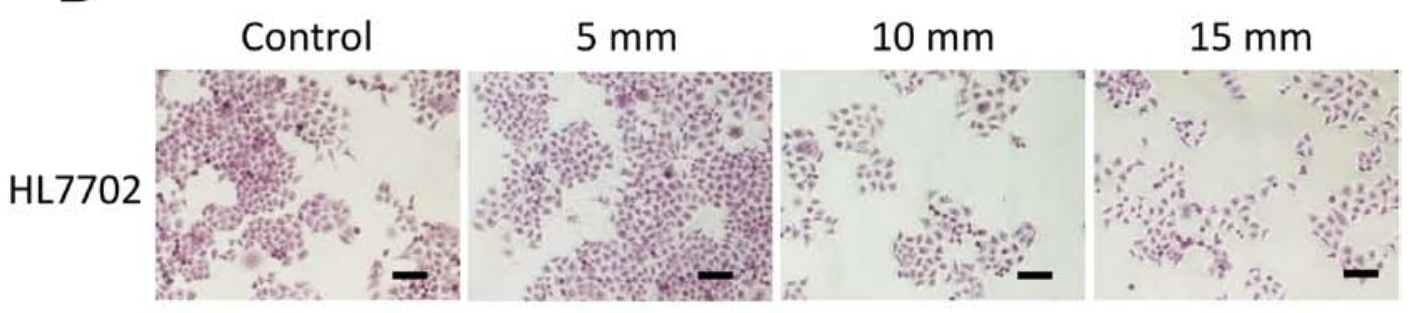

C

HepG2

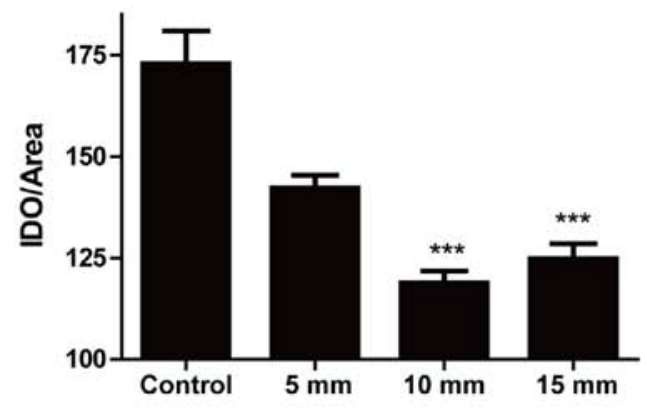

$E$

HepG2

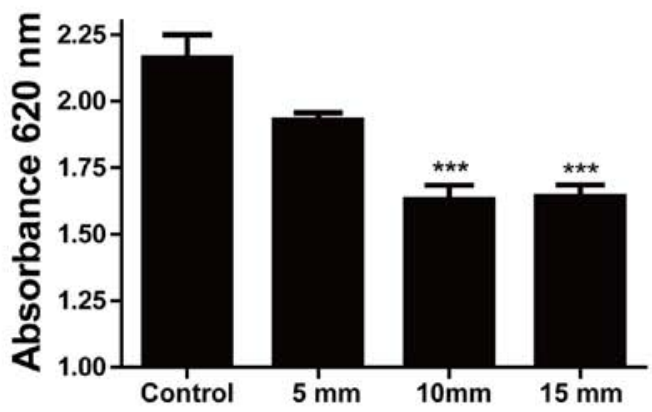

HL-7702

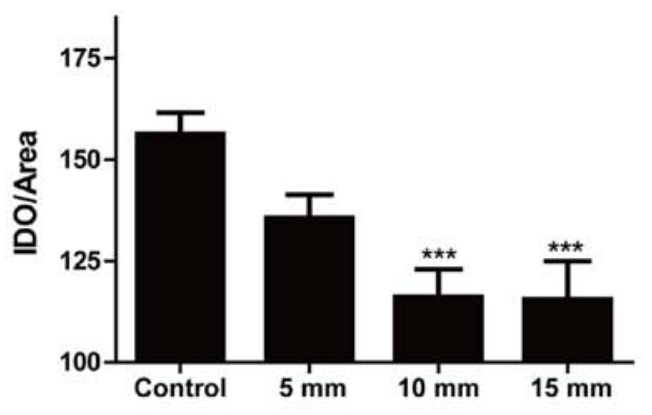

$\mathrm{F}$

HL-7702

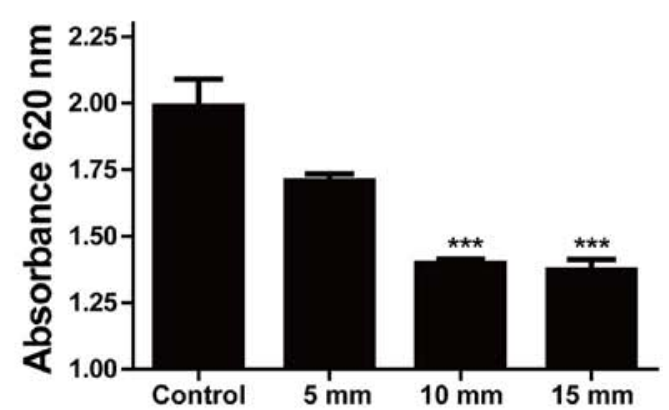

Figure 1. Increasing pressure decreases glycogen concentration in HepG2 and HL-7702 cells. Periodic acid-Schiff analysis of the glycogen concentration in (A) HepG2 and (B) HL-7702 cells treated with different pressures [Control (0), 5, 10 and $15 \mathrm{mmHg}$ (magnification, x100; scale bar, 60 $\mu \mathrm{m}$ ). Quantification of glycogen staining in (C) HepG2 and (D) HL-7702 cells. Glycogen concentration analysis of (E) HepG2 and (F) HL-7702 cells treated with different pressures $(0,5,10$ and $15 \mathrm{mmHg})$. Data are presented as the mean \pm standard error of the mean, $\mathrm{n}=3$. ${ }^{* * *} \mathrm{P}<0.001 \mathrm{vs}$. control. IDO, integrated option density; mm, mmHg.

remained unchanged (Fig. 3A and $\mathrm{B}$ ). It has been reported that p-GYS1 (S641) is the activated isoform of the enzyme and is closely associated with glycogen synthesis. Therefore, the protein expression levels of GYS1 and p-GYS1 (S641) were detected using western blotting. The protein expression levels of total GYS1 remained unchanged (Fig. 3C and D); however, p-GYS1 (S641) expression was significantly decreased under $10 \mathrm{mmHg}$ pressure. These results suggested that mechanical pressure suppressed glycogen synthesis by inhibiting the phosphorylation of GYS1. 
A

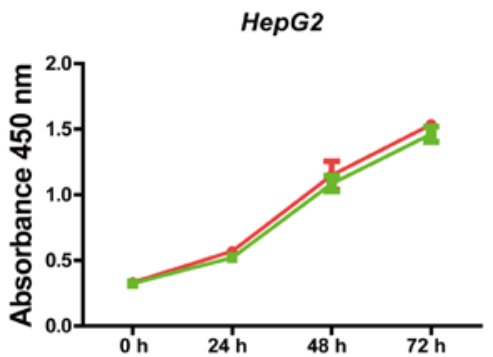

HL-7702

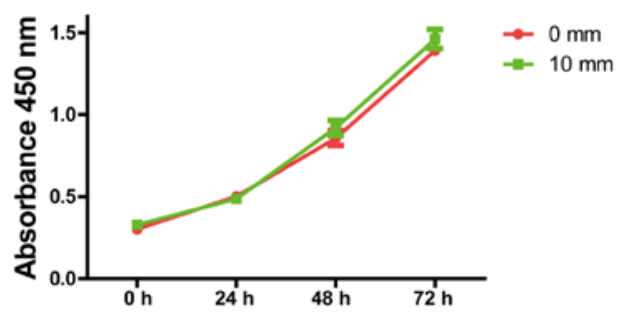

B
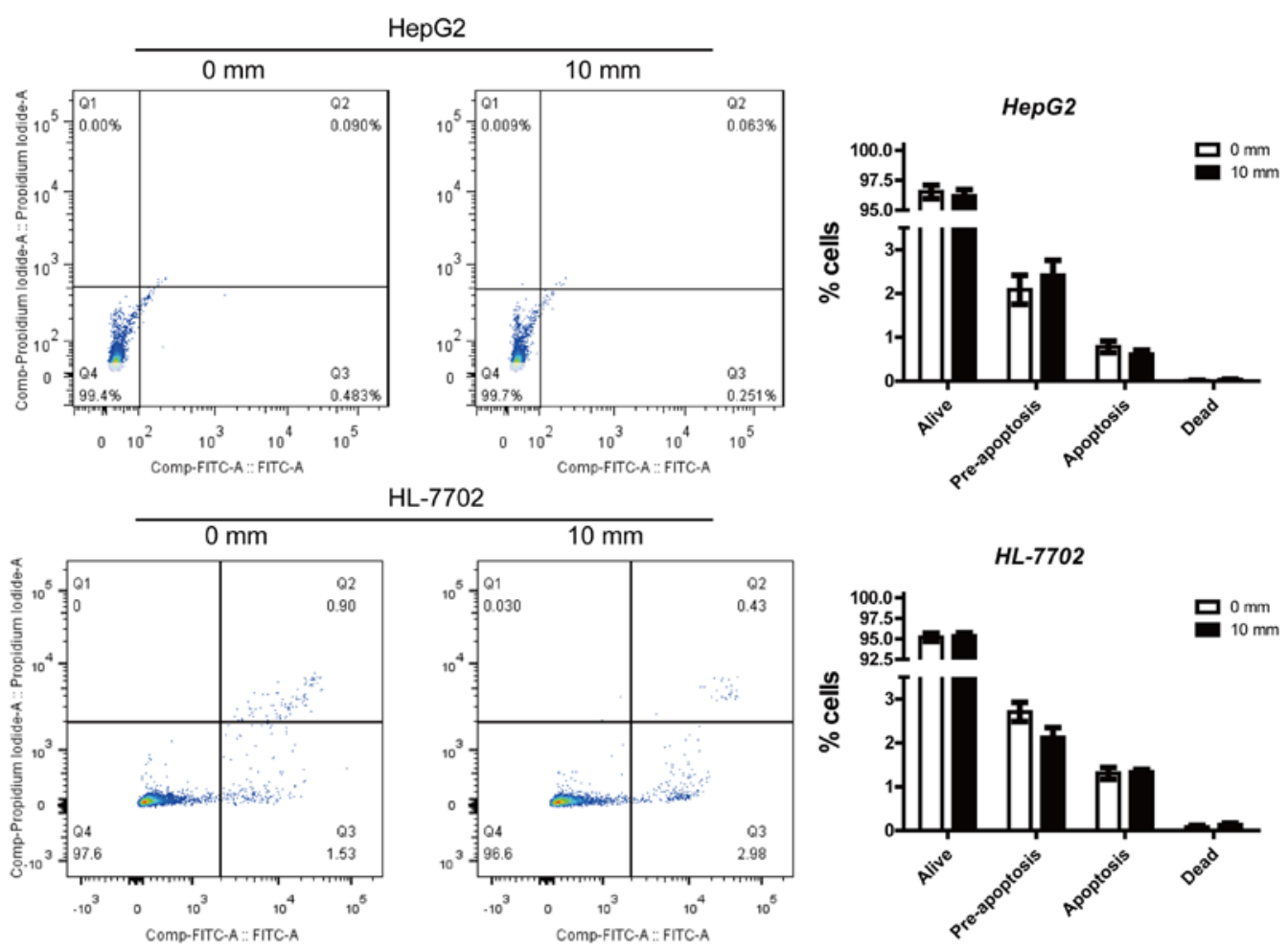

$\mathrm{D}$

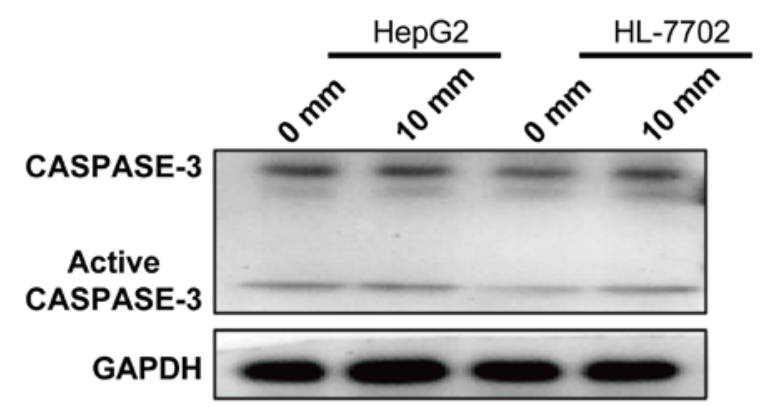

Figure 2. Increasing pressure does not affect cell viability and apoptosis. (A) Cell Counting Kit- 8 analysis of the viability of HepG2 and HL-7702 cells treated with different pressures. Flow cytometric analysis of (B) HepG2 and (C) HL-7702 cells treated with different pressures (0 and 10 mmHg). (D) Western blot analysis of a protein related to apoptosis in HepG2 and HL-7702 cells treated with different pressures $(0 \mathrm{and} 10 \mathrm{mmHg}) . \mathrm{mm}, \mathrm{mmHg}$.

An increase in pressure activates the p53/Pten pathway. To further investigate the mechanisms underlying the effects of pressure exertion on glycogen synthesis, genome-wide gene expression alterations in HepG2 cells treated with different pressures for $24 \mathrm{~h}$ were analyzed using an RNA-seq assay. The gene expression at $10 \mathrm{mmHg}$ was different from that at
$0 \mathrm{mmHg}$; of the 13,456 mapped genes in HepG2 cells, 2,253 were differentially expressed, including 949 downregulated and 1,304 upregulated genes (Fig. 4A). To identify the effects on cell signaling, the data were further analyzed with GSEA. The expression levels of genes associated with the regulation of glycogen metabolism were not significantly altered (Fig. 4B), 
A

HepG2

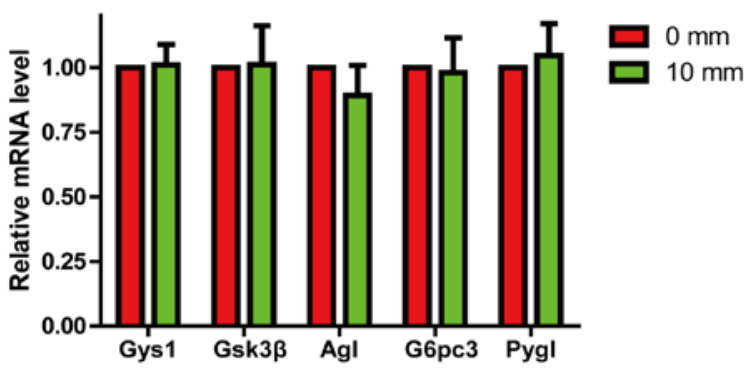

C

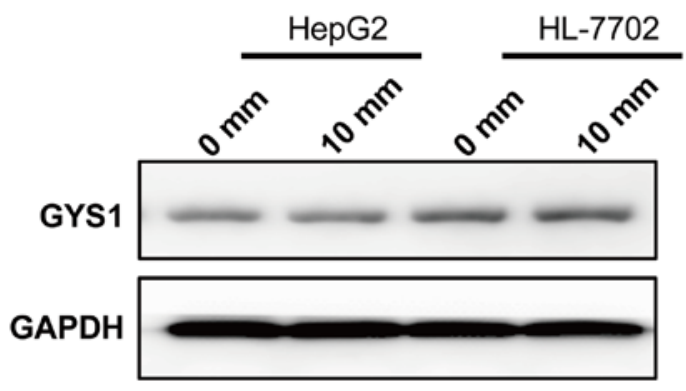

GYS1

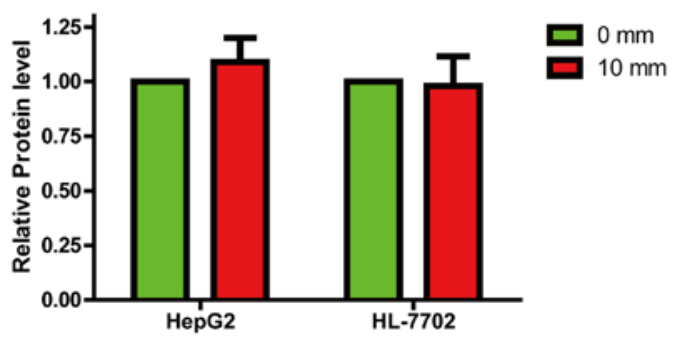

B

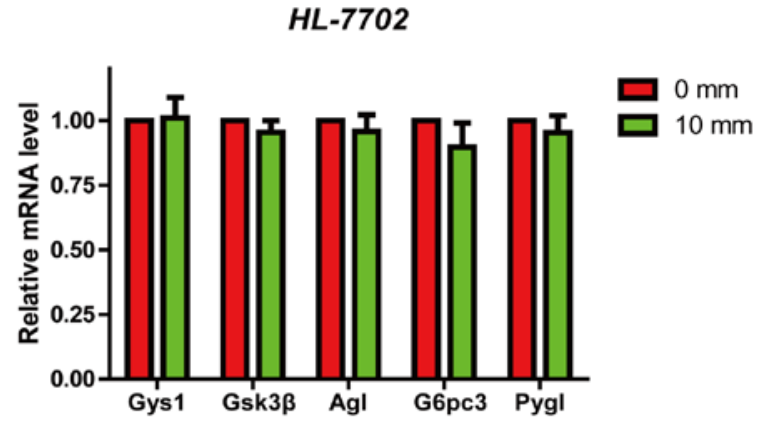

D

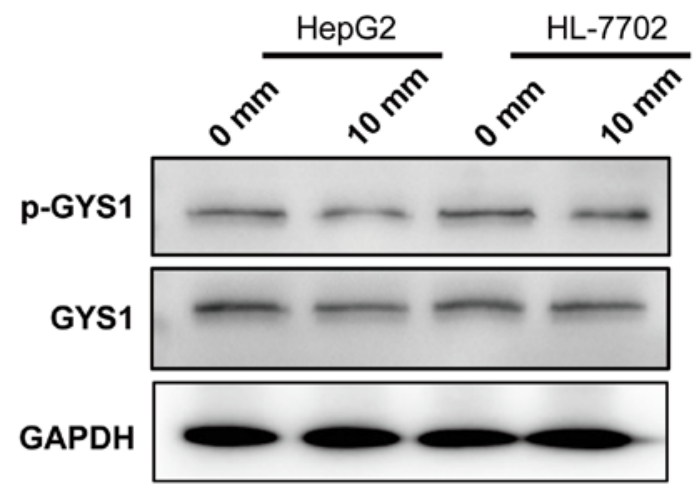

p-GYS1

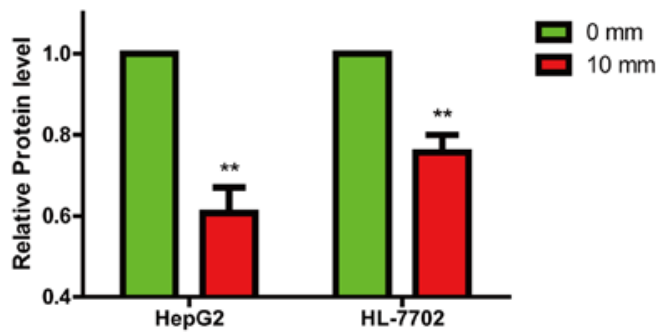

Figure 3. Increasing pressures suppress glycogen synthesis through inhibiting phosphorylation of GYS1. RT-qPCR analysis of the mRNA expression levels of genes related to glycogen synthesis and glycogenolysis in (A) HepG2 and (B) HL-7702 cells treated with different pressures (0 and $10 \mathrm{mmHg}$ ). (C) Western blot analysis of total GYS1 in HepG2 and HL-770 cells treated with different pressures (0 and $10 \mathrm{mmHg}$ ). (D) Western blot analysis of p-GYS1 in HepG2 and HL-770 cells treated with different pressures $(0$ and $10 \mathrm{mmHg})$. GAPDH was used as a loading control. RT-qPCR data are displayed relative to controls as the means \pm standard error of the mean $(\mathrm{n}=3)$. Data are presented as the mean \pm standard error of the mean, $\mathrm{n}=3 .{ }^{* * *} \mathrm{P}<0.01$ vs. control. Agl, amylo- $\alpha-1$, 6-glucosidase, 4-alpha-glucanotransferase; G6pc3, glucose-6-phosphatase catalytic subunit 3; Gys1, glycogen synthase 1; Pygl, glycogen phosphorylase L; $\mathrm{mm}, \mathrm{mmHg}$; RT-qPCR, reverse transcription-quantitative PCR.

consistent with the aforementioned results (Fig. 3A and B). Notably, genes associated with the regulation of apoptosis were significantly upregulated (Fig. 4C). The expression of p53 and Pten were also markedly increased (Fig. 4D). These data indicated that increased pressure inhibited hepatocellular glycogen synthesis by activating the p53/Pten pathway.

An increase in pressure suppresses glycogen synthesis through activation of the p53/Pten pathway. It has been reported that Pten and p53 regulate glycogen synthesis by interacting with the Akt/GSK3 $\beta$ signaling pathway (27). Therefore, the expression levels of p53, Pten and their downstream gene Akt were detected in HepG2 and HL-7702 cells, which were previously incubated under 0 and $10 \mathrm{mmHg}$ pressure for $24 \mathrm{~h}$. When pressure was increased, the mRNA expression levels of p53 and Pten were also increased; however, Akt expression was not significantly altered (Fig. 5A and B). A similar effect was observed in in the resultant protein expression levels (Fig. 5C and D). Notably, p53 regulates Akt activity by inhibiting its phosphorylation at Ser473; in this study, p-Akt was significantly reduced in response to $10 \mathrm{mmHg}$ pressure (Fig. 5D). These data confirmed that pressure may influence glycogen synthesis by regulating the p53/Pten pathway. 
A

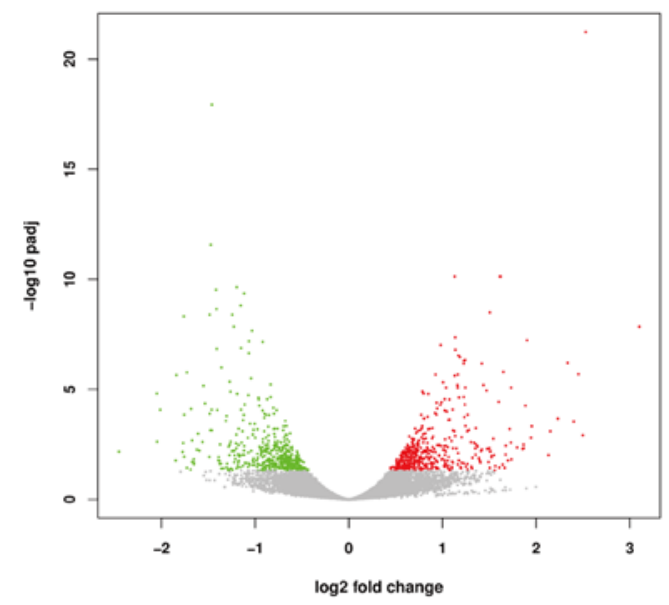

$10 \mathrm{~mm}$ vs. $0 \mathrm{~mm}$

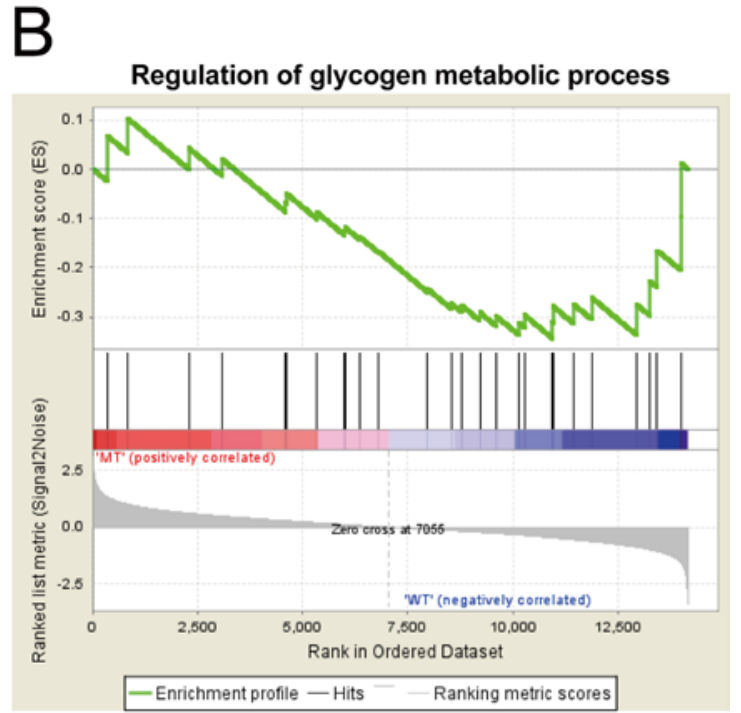

$10 \mathrm{~mm}$ vs. $0 \mathrm{~mm}$
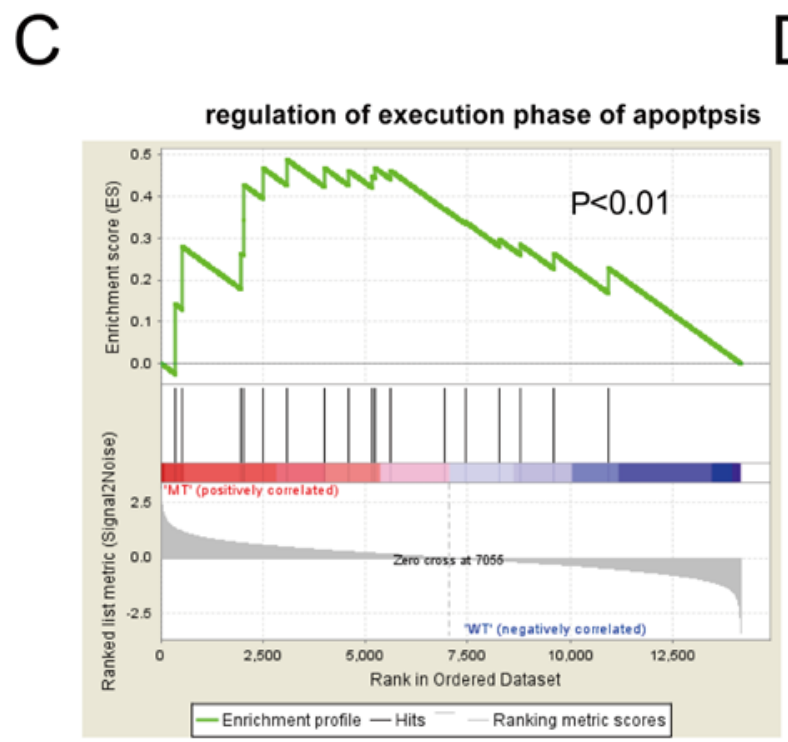

$10 \mathrm{~mm}$ vs. $0 \mathrm{~mm}$

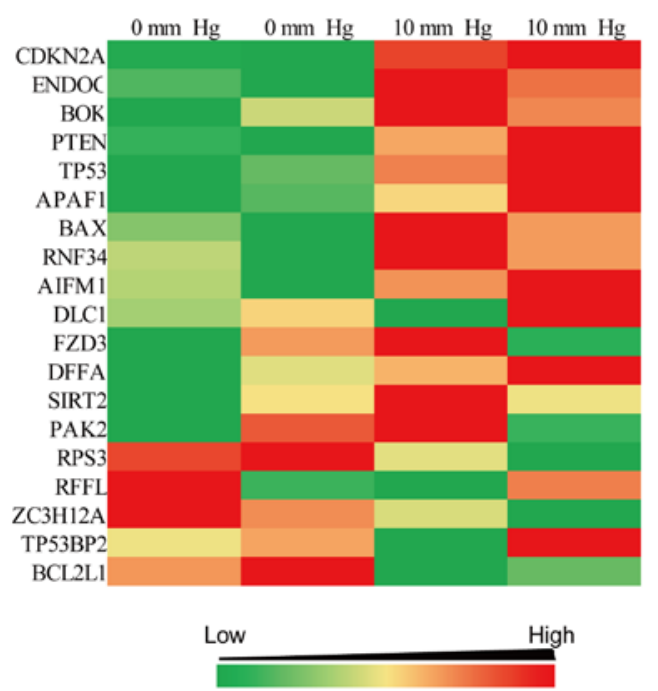

Figure 4. Increasing pressures activate the p53 pathway. (A) Volcano plot of gene expression analysis. Plots of $\log$ P-value versus $\log 2$ fold change for HepG2 cells treated with different pressures $\left(0\right.$ and $10 \mathrm{mmHg}$ ) are shown. The $\mathrm{x}$-axis indicates fold change; the $y$-axis indicates the-log ${ }_{10}$ padj. Red and green genes indicate significantly upregulated and downregulated genes, respectively. (B) GSEA of genes related to glycogen metabolic processes in HepG2 cells treated with different pressures $(0$ and $10 \mathrm{mmHg}$ ). (C) GSEA of genes related to the execution phase of apoptosis in HepG2 cells treated with different pressures $(0$ and $10 \mathrm{mmHg}$ ). (D) Heat map analysis of the genes related to the execution phase of apoptosis in HepG2 cells treated with different pressures $(0$ and $10 \mathrm{mmHg})$. mm, mmHg; GSEA, gene set enrichment analysis; padj, adjusted P-value.

\section{Discussion}

The regulation of hepatocellular glycogen synthesis and glycogenolysis is one of the principle methods in the maintenance of blood glucose concentration. The effects of portal hypertension on hepatocellular glycogen metabolism remain unclear. The present study demonstrated that an increase in extracellular pressure significantly inhibited hepatocellular glycogen synthesis and GYS1 activity. Further experiments demonstrated that these effects were closely associated with the p53/Pten pathway. This pathway inhibits Akt signaling and glycogen synthesis (Fig. 5E). Therefore, the present study revealed an association between mechanical pressure and hepatocyte metabolism.
Mechanical factors, including pressure, influence the extracellular microenvironment. It has been reported that such factors regulate cellular proliferation and differentiation. Numerous studies have demonstrated that mechanical force, such as pressure and stretch, is an important regulator in cell metabolism (28-30). The present study suggested that portal hypertension significantly inhibited the synthesis of hepatocellular glycogen. It has been reported that the right atrial pressure of patients with portal hypertension has an interquartile range of $12.0-15.5 \mathrm{mmHg}(11,31)$. The gradient pressures detected in this study were $<15.5 \mathrm{mmHg}$; therefore, it was hypothesized that this study could partially reflect the pressure environment in vivo. Additionally, it 
A

B

HepG2

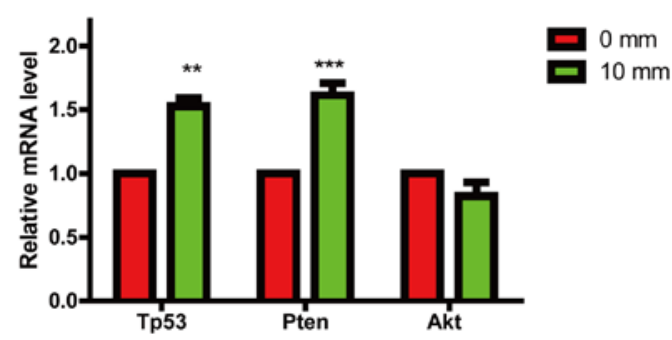

HL-7702

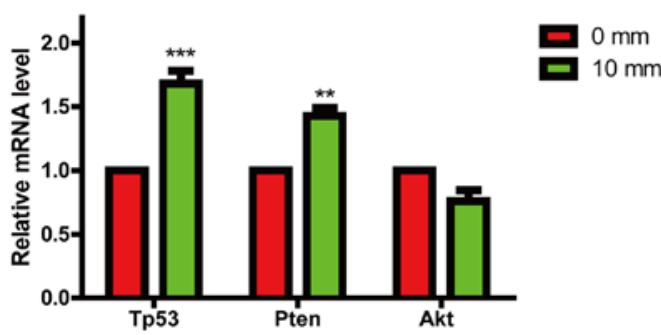

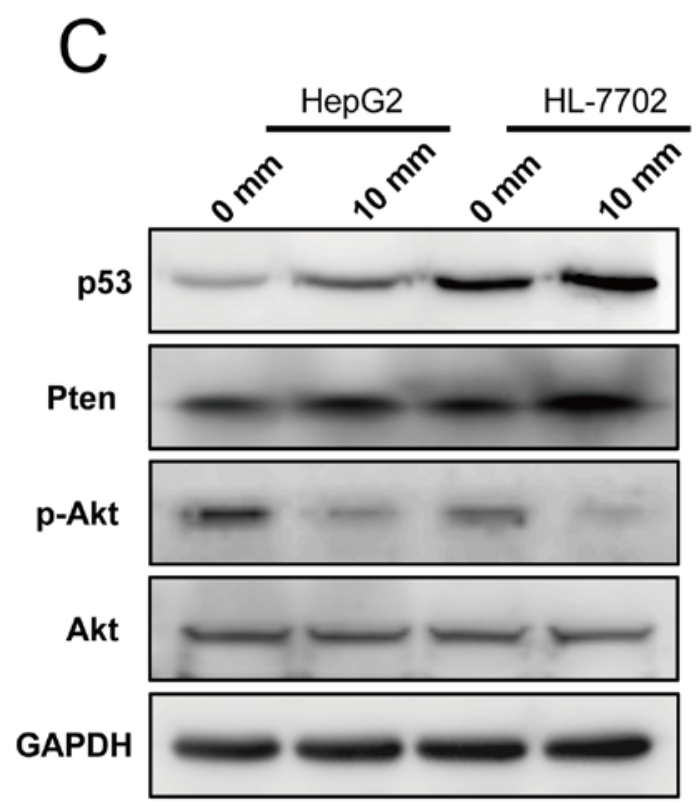

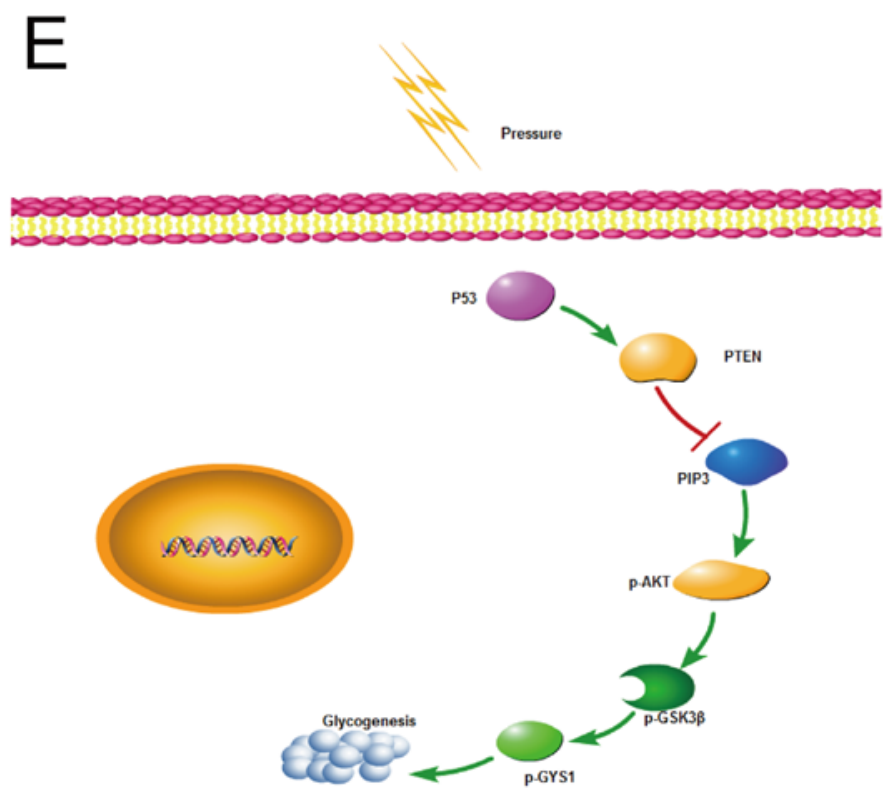

D
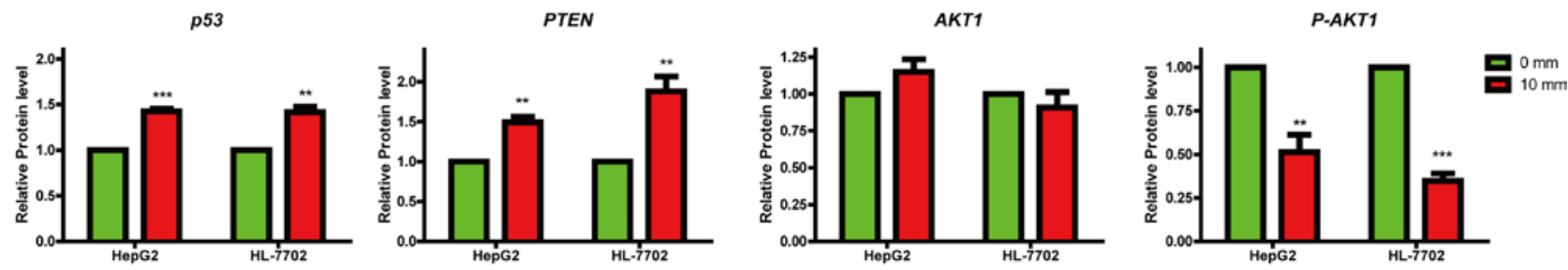

Figure 5. Increasing pressures suppress glycogen synthesis through activating the p53 pathway. RT-qPCR analysis of the mRNA expression levels of genes related to the p53 pathway in (A) HepG2 and (B) HL-770 cells treated with different pressures ( 0 and $10 \mathrm{mmHg}$ ). RT-qPCR data are displayed relative to controls as the mean \pm standard error of the mean $(n=3)$. (C and D) Western blot analysis of proteins related to the p53 pathway in HepG2 and HL-7702 cells treated with different pressures $(0$ and $10 \mathrm{mmHg}$ ). (E) Proposed schematic diagram of the regulatory role of pressure in hepatocellular glycogen synthesis. Data are presented as the mean \pm standard error of the mean, $\mathrm{n}=3 .{ }^{* * *} \mathrm{P}<0.01,{ }^{* * * *} \mathrm{P}<0.001$ vs. control. Akt, protein kinase B; GYS1, glycogen synthase $1 ; \mathrm{mm}, \mathrm{mmHg}$; P, phosphorylated; PIP3, phosphatidylinositol (3,4,5)-trisphosphate; Pten, phosphatase and tensin homolog; RT-qPCR, reverse transcription-quantitative PCR; $\mathrm{mm}, \mathrm{mmHg}$.

was previously demonstrated that the plasma glucagon levels of patients with portal hypertension are particularly unstable (32). The presented data suggested that the influence of portal hypertension on glycogen synthesis may provide a reason for this instability. Muscle is another important organ in glycogen metabolism and is frequently stimulated by mechanical forces $(33,34)$. Therefore, further studies focusing on the role of these mechanical forces, including stress and tension in the muscle, glycogen synthesis and glycogenolysis, are required. 
The liver is the primary organ involved in the regulation of blood glucose, and glycogen synthesis and glycogenolysis are key regulatory processes. Previous studies have demonstrated that glycogen synthesis is regulated by chemical signals, including hormones and small molecules $(35,36)$. In the present study, it was established that pressure served an important regulatory role in glycogen synthesis. Furthermore, previous studies have confirmed that glycogen synthesis is tightly regulated by intracellular and extracellular microenvironmental signals $(37,38)$. The Akt/GSK3 $\beta$ pathway is a critical component of hepatocellular glycogen synthesis; notably, insulin promotes the synthesis of glycogen by activating this pathway (37). The present study revealed that extracellular pressure inhibited this pathway, and regulated the expression of associated genes. In addition to glycogen synthesis and glycogenolysis, the liver also facilitates gluconeogenesis and lipid metabolism, and previous studies have demonstrated that the Akt1/GSK3 $\beta$ pathway is also critically involved in gluconeogenesis and lipid metabolism $(39,40)$. These data suggested that portal hypertension may also serve a principal role in gluconeogenesis and lipid metabolism.

The p53 pathway is frequently activated in response to external stimuli, and chemotherapy-induced apoptosis is caused by p53 activation (41). The present study revealed that extracellular pressure induced upregulation of p53 mRNA expression, inducing the expression of its downstream gene, Pten. These results suggested that activation of the p53 pathway may be one of the ways in which cells respond to external stimuli. The p53 pathway regulates the metabolism of cellular glucose and lipids $(42,43)$; these studies further demonstrated that when cells are exposed to external stimuli at a low-intensity, p53 may be upregulated, and subsequently inhibit glucose metabolism. Therefore, the p53 pathway may serve a prominent role in the metabolic response to external stimuli.

Despite these promising results, several questions remain unanswered. Firstly, it is intriguing that the glycogen concentration in the $15 \mathrm{mmHg}$ pressure group was not considerably decreased compared with in the $10 \mathrm{mmHg}$ group. The reason for this could be that $15 \mathrm{mmHg}$ pressure may reduce glycogen concentration compared to $10 \mathrm{mmHg}$; however, the difference may be too small and therefore could not be detected. Secondly, this study indicated that p53 is a critical gene that functions as a sensor that may respond to mechanical pressure. However, further work is required to reveal the direct relationship between pressure and $\mathrm{p} 53$. In addition, this study demonstrated the effects of pressure on HepG2 and HL-7702 cell lines; further research should be undertaken to reveal the physiological role of pressure in primary hepatocytes. Genes associated with the regulation of apoptosis were significantly upregulated; however, cell apoptosis was not affected by mechanical pressure treatment for $24 \mathrm{~h}$. One reason for this may be that the alterations in the expression of apoptosis-associated genes are insufficient to influence cell apoptosis in a short period of time. Finally, the results revealed that pressure did not affect cell viability and apoptosis in vitro. A further study focusing on the effects of pressure on cell viability and apoptosis, as well as glycogen metabolism, in vivo is therefore suggested.

To the best of our knowledge, the present study is the first to demonstrate that extracellular pressure significantly inhibited hepatocellular glycogen synthesis. It was further revealed that the p53 pathway was involved in the regulation of hepatocellular glycogen synthesis. These results not only suggested a regulatory effect for mechanical pressure in hepatocellular carbohydrate metabolism, but also implied its role in hepatocellular lipid. Given the current challenges of portal hypertension treatment, the present study, and the associated questions raised, demonstrated the requirement for further investigation into the role of pressure in hepatocytes, and the potential associated molecular mechanisms.

\section{Acknowledgements}

Not applicable.

\section{Funding}

This study was supported by the National Nature Science Foundation of China (grant nos. NSFC-11602295 and NSFC-11472300).

\section{Availability of data and materials}

All data generated or analyzed during this study are included in this published article.

\section{Authors' contributions}

LZ and JC were involved in study conception and design, manuscript revision, funding support and study supervision. JS performed the experiments, data analysis and manuscript writing. YS performed data analysis and manuscript writing. SS and XL performed statistical analysis. All authors read and approved the final manuscript.

\section{Ethics approval and consent to participate}

Not applicable.

\section{Patient consent for publication}

Not applicable.

\section{Competing interests}

The authors declare that they have no competing interests.

\section{References}

1. Nestor-Bergmann A, Goddard G and Woolner S: Force and the spindle: Mechanical cues in mitotic spindle orientation. Semin Cell Dev Biol 34: 133-139, 2014.

2. Lesman A, Notbohm J, Tirrell DA and Ravichandran G: Contractile forces regulate cell division in three-dimensional environments. J Cell Biol 205: 155-162, 2014.

3. Harada S and Rodan GA: Control of osteoblast function and regulation of bone mass. Nature 423: 349-355, 2003.

4. Robling AG, Castillo AB and Turner $\mathrm{CH}$ : Biomechanical and molecular regulation of bone remodeling. Annu Rev Biomed Eng 8: 455-498, 2006.

5. Bin G, Cuifang W, Bo Z, Jing W, Jin J, Xiaoyi T, Cong C, Yonggang $C$, Liping A, Jinglin $M$ and Yayi X: Fluid shear stress inhibits TNF- $\alpha$-induced osteoblast apoptosis via ERK5 signaling pathway. Biochem Biophys Res Commun 466: $117-123,2015$. 
6. Caiazzo M, Okawa Y, Ranga A, Piersigilli A, Tabata Y and Lutolf MP: Defined three-dimensional microenvironments boost induction of pluripotency. Nat Mater 15: 344-352, 2016.

7. Altman GH, Horan RL, Martin I, Farhadi J, Stark PR, Volloch V, Richmond JC, Vunjak-Novakovic G and Kaplan DL: Cell differentiation by mechanical stress. ASEB J 16: 270-272, 2002.

8. Estes BT, Gimble JM and Guilak F: Mechanical signals as regulators of stem cell fate. Curr Top Dev Biol 60: 91-126, 2004.

9. Pillai AK, Andring B, Patel A, Trimmer C and Kalva SP: Portal hypertension: A review of portosystemic collateral pathways and endovascular interventions. Clin Radiol 70: 1047-1059, 2015.

10. Berzigotti A, Seijo S, Reverter E and Bosch J: Assessing portal hypertension in liver diseases. Expert Rev Gastroenterol Hepatol 7: 141-155, 2013.

11. Takuma Y, Nouso K, Morimoto Y, Tomokuni J, Sahara A, Takabatake H, Matsueda K and Yamamoto H: Portal hypertension in patients with liver cirrhosis: Diagnostic accuracy of spleen stiffness. Radiology 279: 609-619, 2016.

12. Bloom S, Kemp W and Lubel J: Portal hypertension: Pathophysiology, diagnosis and management. Intern Med J 45: 16-26, 2015.

13. Ksiazyk J, Lyszkowska M and Kierkus J: Energy metabolism in portal hypertension in children. Nutrition 12: 469-474, 1996.

14. Arias N, Méndez M, Arias J and Arias JL: Brain metabolism and spatial memory are affected by portal hypertension. Metab Brain Dis 27: 183-191, 2012.

15. Mohan P and Venkataraman J: Minimal hepatic encephalopathy in noncirrhotic portal hypertension. Eur J Gastroenterol Hepatol 23: 194-195, 2011.

16. Vogels BA, van Steynen B, Maas MA, Jörning GG and Chamuleau RA: The effects of ammonia and portal-systemic shunting on brain metabolism, neurotransmission and intracranial hypertension in hyperammonaemia-induced encephalopathy. J Hepatol 26: 387-395, 1997.

17. Perisic M, Ilic-Mostic T, Stojkovic M, Culafic D and Sarenac R: Doppler hemodynamic study in portal hypertension and hepatic encephalopathy. Hepatogastroenterology 52: 156-160, 2005.

18. Petersen MC, Vatner DF and Shulman GI: Regulation of hepatic glucose metabolism in health and disease. Nat Rev Endocrinol 13: 572-587, 2017

19. Boden G, Cheung P, Stein TP, Kresge K and Mozzoli M: FFA cause hepatic insulin resistance by inhibiting insulin suppression of glycogenolysis. Am J Physiol Endocrinol Metab 283: E12-E19, 2002.

20. Yang WM, Jeong HJ, Park SY and Lee W: Saturated fatty acid-induced miR-195 impairs insulin signaling and glycogen metabolism in HepG2 cells. FEBS Lett 588: 3939-3946, 2014.

21. Kir S, Beddow SA, Samuel VT, Miller P, Previs SF, Suino-Powell K, Xu HE, Shulman GI, Kliewer SA and Mangelsdorf DJ: FGF19 as a postprandial, insulin-independent activator of hepatic protein and glycogen synthesis. Science 331: 1621-1624, 2011.

22. Liu TY, Shi CX, Gao R, Sun HJ, Xiong XQ, Ding L, Chen Q, Li YH, Wang JJ, Kang YM and Zhu GQ: Irisin inhibits hepatic gluconeogenesis and increases glycogen synthesis via the PI3K/Akt pathway in type 2 diabetic mice and hepatocytes. Clin Sci (Lond) 129: 839-850, 2015.

23. Prats C, Graham TE and Shearer J: The dynamic life of the glycogen granule. J Biol Chem 293: 7089-7098, 2018.

24. Watanabe S, Nagashio Y, Asaumi H, Nomiyama Y, Taguchi M, Tashiro M, Kihara Y, Nakamura $\mathrm{H}$ and Otsuki M: Pressure activates rat pancreatic stellate cells. Am J Physiol Gastrointest Liver Physiol 287: G1175-G1181, 2004.

25. Wu HJ, Zhang ZQ, Yu B, Liu S, Qin KR and Zhu L: Pressure activates Src-dependent FAK-Akt and ERK1/2 signaling pathways in rat hepatic stellate cells. Cell Physiol Biochem 26: 273-280, 2010.

26. Livak KJ and Schmittgen TD: Analysis of relative gene expression data using real-time quantitative PCR and the 2(-Delta Delta C(T)) method. Methods 25: 402-408, 2001.
27. Fang W, Guo J, Cao Y, Wang S, Pang C, Li M, Dou L, Man Y, Huang X, Shen T and Li J: MicroRNA-20a-5p contributes to hepatic glycogen synthesis through targeting p63 to regulate p53 and PTEN expression. J Cell Mol Med 20: 1467-1480, 2016.

28. Isogai T, Park JS and Danuser G: Cell forces meet cell metabolism. Nat Cell Biol 19: 591-593, 2017.

29. Dziegala M, Kobak KA, Kasztura M, Bania J, Josiak K, Banasiak W, Ponikowski P and Jankowska EA: Iron depletion affects genes encoding mitochondrial electron transport chain and genes of non-oxidative metabolism, pyruvate kinase and lactate dehydrogenase, in primary human cardiac myocytes cultured upon mechanical stretch. Cells 7: pii: E175, 2018.

30. Yi SH, Zhang Y, Tang D and Zhu L: Mechanical force and tensile strain activated hepatic stellate cells and inhibited retinol metabolism. Biotechnol Lett 37: 1141-1152, 2015.

31. Denault AY, Beaubien-Souligny W, Elmi-Sarabi M, Eljaiek R, El-Hamamsy I, Lamarche Y, Chronopoulos A, Lambert J, Bouchard J and Desjardins G: Clinical significance of portal hypertension diagnosed with bedside ultrasound after cardiac surgery. Anesth Analg 124: 1109-1115, 2017.

32. Johnson TJ, Quigley EM, Adrian TE, Jin G and Rikkers LF: Glucagon, stress, and portal hypertension. Plasma glucagon levels and portal hypertension in relation to anesthesia and surgical stress. Dig Dis Sci 40: 1816-1823, 1995.

33. Mohamed JS, Lopez MA and Boriek AM: Mechanical stretch up-regulates microRNA-26a and induces human airway smooth muscle hypertrophy by suppressing glycogen synthase kinase- $3 \beta$. J Biol Chem 285: 29336-29347, 2010.

34. Ørtenblad N, Westerblad H and Nielsen J: Muscle glycogen stores and fatigue. J Physiol 591: 4405-4413, 2013.

35. Roach PJ, Depaoli-Roach AA, Hurley TD and Tagliabracci VS: Glycogen and its metabolism: Some new developments and old themes. Biochem J 441: 763-787, 2012.

36. Nakano K, Takeshita S, Kawasaki N, Miyanaga W, Okamatsu Y, Dohi M and Nakagawa T: AJS1669, a novel small-molecule muscle glycogen synthase activator, improves glucose metabolism and reduces body fat mass in mice. Int J Mol Med 39: 841-850, 2017.

37. Han C, Wei S, He F, Liu D, Wan H, Liu H, Li L, Xu H, Du X and $\mathrm{Xu} F$ : The regulation of lipid deposition by insulin in goose liver cells is mediated by the PI3K-AKT-mTOR signaling pathway. PLoS One 10: e0098759, 2015.

38. Nocito L, Kleckner AS, Yoo EJ, Jones Iv AR, Liesa M and Corkey BE: The extracellular redox state modulates mitochondrial function, gluconeogenesis, and glycogen synthesis in murine hepatocytes. PLoS One 10: e0122818, 2015.

39. Chang YS, Tsai CT, Huangfu CA, Huang WY, Lei HY, Lin CF, Su IJ, Chang WT, Wu PH, Chen YT, et al: ACSL3 and GSK-3 $\beta$ are essential for lipid upregulation induced by endoplasmic reticulum stress in liver cells. J Cell Biochem 112: 881-893, 2011.

40. Venna VR, Benashski SE, Chauhan A and McCullough LD: Inhibition of glycogen synthase kinase- $3 \beta$ enhances cognitive recovery after stroke: The role of TAK1. Learn Mem 22: 336-343, 2015.

41. Hientz K, Mohr A, Bhakta-Guha D and Efferth T: The role of p53 in cancer drug resistance and targeted chemotherapy. Oncotarget 8: 8921-8946, 2017.

42. Jiang P, Du W, Wang X, Mancuso A, Gao X, Wu M and Yang X: p53 regulates biosynthesis through direct inactivation of glucose-6-phosphate dehydrogenase. Nat Cell Biol 13: 310-316, 2011.

43. Liu J, Zhang C, Hu W and Feng Z: Tumor suppressor p53 and its mutants in cancer metabolism. Cancer Lett 356: 197-203, 2015.

This work is licensed under a Creative Commons Attribution-NonCommercial-NoDerivatives 4.0 International (CC BY-NC-ND 4.0) License. 